\title{
Reverse Impaired Fasting Glucose in Overweight and Obese Patients Participating in a Self-Paid University Weight Management Program
}

\author{
Vijaya Surampudi ${ }^{1}$, XinKai Zhou ${ }^{1}$, Chi-Hong Tseng ${ }^{1}$, David Heber ${ }^{1}$, and Zhaoping Li $^{1}$ \\ ${ }^{1} \mathrm{UCLA}$
}

February 24, 2021

\begin{abstract}
Aims: The progression of prediabetes to T2DM can be delayed through diet modification and weight management. This retrospective review examines the impact on blood sugar, blood pressure, and fasting lipids of a self-pay weight management program utilizing protein-enriched meal replacements and partial meal replacement diets providing $2.2 \mathrm{grams} / \mathrm{kg} / \mathrm{day}$ of protein in overweight and obese patients with impaired fasting glucose and excess body fat (average \%body fat ca. 40\%). Methods: The medical records of 4634 obese patient who participated in the self-pay UCLA Weight Management Program were reviewed to identify 2572 eligible patients for this retrospective study of the impact of weight loss over 3 months on patients with normal fasting glucose (NFG) ( $\mathrm{n}=1396)$ or impaired fasting glucose (IFG) $(\mathrm{n}=1176)$. Results: Patients with IFG lost comparable amounts of weight (ca. $10 \mathrm{~kg}$ ) at three months as did the subjects with NFG. Fasting blood glucose in the IFG group decreased from $108.49 \pm 6.4 \mathrm{mg} / \mathrm{dl}$ to $101.8 \pm 9.41 \mathrm{mg} / \mathrm{dl}(\mathrm{p}<0.0001)$ after three months. There were also significant reductions in triglycerides, and both systolic and diastolic blood pressure in both groups in association with weight loss. Conclusion: Our medically supervised self-pay multidisciplinary weight management program utilizing protein-enriched diets and meal replacements reduced fasting blood glucose levels in patients with IFG while reducing triglycerides and blood pressure in all patients over three months.
\end{abstract}

Title: Reverse Impaired Fasting Glucose in Overweight and Obese Patients Participating in a Self-Paid University Weight Management Program

Authors: Vijaya Surampudi $\mathrm{MD}^{1}$, MS, Xinkai Zhou MS ${ }^{1}$, Chi-Hong Tseng $\mathrm{PhD}^{1}$, David Heber MD, $\mathrm{PhD}^{1}$, Zhaoping Li MD, $\mathrm{PhD}^{1,2}$

Affiliation:

${ }^{1}$ Department of Medicine, University of California Los Angeles

${ }^{2}$ Department of Medicine, VA Greater Los Angeles Health Care System

Disclosure: The authors report no conflict of interest.

Tables: 1

Figures: 3

Key terms: Obesity, impaired fasting glucose, prediabetes, weight loss, personalized weight management Corresponding author:

Zhaoping Li 
zli@mednet.ucla.edu

1000 Veteran Ave A670

Los Angeles, California 90024

United States

\section{Abstract}

Aims: The progression of prediabetes to T2DM can be delayed through diet modification and weight management. This retrospective review examines the impact on blood sugar, blood pressure, and fasting lipids of a self-pay weight management program utilizing protein-enriched meal replacements and partial meal replacement diets providing 2.2 grams $/ \mathrm{kg} /$ day of protein in overweight and obese patients with impaired fasting glucose and excess body fat (average \%body fat ca. 40\%).

Methods: The medical records of 4634 obese patient who participated in the self-pay UCLA Weight Management Program were reviewed to identify 2572 eligible patients for this retrospective study of the impact of weight loss over 3 months on patients with normal fasting glucose (NFG) (n=1396) or impaired fasting glucose (IFG) $(\mathrm{n}=1176)$.

Results: Patients with IFG lost comparable amounts of weight (ca. $10 \mathrm{~kg}$ ) at three months as did the subjects with NFG. Fasting blood glucose in the IFG group decreased from $108.49 \pm 6.4 \mathrm{mg} / \mathrm{dl}$ to $101.8 \pm 9.41 \mathrm{mg} / \mathrm{dl}$ $(\mathrm{p}<0.0001)$ after three months. There were also significant reductions in triglycerides, and both systolic and diastolic blood pressure in both groups in association with weight loss.

Conclusion: Our medically supervised self-pay multidisciplinary weight management program utilizing protein-enriched diets and meal replacements reduced fasting blood glucose levels in patients with IFG while reducing triglycerides and blood pressure in all patients over three months.

\section{Introduction}

Excess body fat especially intra-abdominal fat is central to the progression of prediabetes to Type 2 Diabetes Mellitus (T2DM). One measure of impaired glucose tolerance is impaired fasting glucose (IFG) defined a fasting blood sugar $>100 \mathrm{mg} / \mathrm{dl}$. The Center for Disease Control (CDC) estimates 86 million or more than a third of the U.S. population to have prediabetes (1) which has been observed to progress to T2DM at a rate of $5-10 \%$ annually (2). The prevalence of prediabetes is projected to exceed 470 million worldwide by 2035. Obesity and prediabetes have also been associated with hypertension, lipid disorders, obstructive sleep apnea, fatty liver disease and cancer (3). Microvascular changes have been identified prior to the development of T2DM are including nephropathy, neuropathy, erectile dysfunction, and retinopathy (4). Prediabetes and obesity are also associated with an increased risk for cardiovascular diseases (5, 6). A continuous inverse correlation between glucose levels and cognitive test results in the absence of diabetes has been demonstrated in cross-sectional population studies (7).

The most recent Endocrine Society clinical practice guidelines for the primary prevention of atherosclerotic cardiovascular disease (ASCVD) and T2DM in patients at metabolic risk recommend prescribing lifestyle modification to reduce blood glucose prior to instituting pharmacological treatment (8). These recommendations are based on several hallmark trials including the U.S. Diabetes Prevention Program (DPP) and Look AHEAD (Action for Health in Diabetes) study. The U.S. Diabetes Prevention Program (DPP) reported that lifestyle modification resulted in a 58 percent reduction in the incidence of T2DM and was more effective than metformin (9). The Da Qing Diabetes Prevention Outcome Study in China demonstrated that lifestyle intervention in people with impaired glucose tolerance delayed the onset of T2DM and reduced the incidence of cardiovascular events, microvascular complications, and all-cause mortality (10).

This retrospective study analyzed the clinic records of patients enrolled in the UCLA Medical Weight Management program which utilizes a unique prescriptive protein-enriched low calorie and very low calorie diets in combination with supportive behavioral groups, medical and dietetic supervision on an individualized basis. 
Protein prescriptions were based on lean body mass assessments using bioelectrical impedance analysis by providing 1 gram per pound of lean body mass per day which averaged about 2 grams per kg body weight per day.

\section{Methods}

\section{Subjects}

This was a retrospective study of 4634 patients who participated in the UCLA Weight Management Program (also called the UCLA Risk Factor Obesity Program) between 1991 and 2016. The study consisting of a retrospective review of medical records retained by the program and was approved by the Institutional Review Board of University of California, Los Angeles. Patient records were included in the analysis if patients met the following inclusion criteria: 1) $>18$ years of age; 2) overweight or obese with a BMI $>25$ $\mathrm{kg} / \mathrm{m} 2 ; 3)$ enrolled in the program for the first time; 4) participated in the program weekly without missing more than three consecutive weekly visits during the three-month period. Patients with diagnosed T2DM based on a fasting blood glucose $>126 \mathrm{mg} / \mathrm{dl}$ or taking hypoglycemic medications were excluded from study.

\section{Clinical Program}

The UCLA Medical Weight Management Program focuses on individualized dietary prescriptions based on lean body mass assessed by bioimpedance analysis (BIA). Typical meal plans included between 5-10 meal replacements/day and a total of between 500 and 1000 Calories per day designed to achieved a deficit of at least 500 Calories per day below maintenance calories. Most patients began the program with total meal replacement while some selected a modified plan with one whole food meal per day. Prescribed calories from meal replacements and food were based on calorie deficits below the resting metabolic rate estimated from lean body mass. Patients were prescribed dietary protein intakes match to the lean body mass at 1 gram per pound lean body mass per day leading to a total protein intake of approximately $2.2 \mathrm{grams}$ of protein $/ \mathrm{kg}$ body weight per day. Meal replacements from several suppliers utilized high quality protein combined with $40 \%$ carbohydrate vitamins and minerals. The proteins used included soy, pea, casein and whey proteins. After a period of time, many patients transitioned to a partial meal replacement program where four or five servings of meal replacements per day combined with a defined meal of about 300 calories of lean protein and non-starchy vegetables. After the first 2 weeks in the program, patients were encouraged to exercise for 30 min per day and to add resistance exercise.

Weights and vital signs were measured weekly. Laboratory tests including complete blood count, electrolytes, and a basic metabolic panel (Quest Diagnostics, San Juan Capistrano, CA) were also recorded at baseline and at 3-week intervals. The BIA to assess lean body mass was repeated every 6-8 weeks.

Exercise was recommended and encouraged in the physician and dietitian visits after the first two weeks in the program. Patient visits at the clinic also included consultations with physicians and dietitians. The program also included voluntary attendance at weekly group behavior change and group nutrition education classes.

The cost of the program varied from $\$ 50$ to $\$ 65$ per week depending on the level of dietary restriction and need for monitoring including meal replacement products. Insurance plans including private insurance and health savings accounts were typically used to cover the cost of the program in part. Payments were collected monthly in advance and were non-refundable unless there was prior notice for planned vacations or family emergencies. Laboratory tests, body composition analysis, and classes were included in the monthly program fee.

\section{Outcome measures}

The primary outcome was amount of weight-loss and fasting blood glucose values after three months. Secondary outcomes included percent body fat, blood pressure and lipid values.

\section{Statistical Analysis}


Patient demographic data and baseline clinical history were summarized using median (IQR) for continuous variables and frequency (percentage) for categorical variables. Continuous variables were compared using the Wilcoxon rank-sum test, whereas categorical variables were compared using the Pearson's chi-squared test. We also summarized the number (percentage) of patients who remained in the study at three months.

Glucose levels were analyzed as a dichotomized variable (IFG) if levels were greater than $100 \mathrm{mg} / \mathrm{dL}$. For the continuous glucose outcome, we used the linear mixed effects model with fixed time and group (baseline IFG status Yes/No) effect and random subject effect to model the trajectory of glucose levels over time. Specifically, time was modeled as piecewise linear with change points at 1 and 3 months. The random effects model takes into account the correlation between the observations within the same subject. Model estimates of glucose levels and their $95 \%$ confidence intervals were summarized for both the IFG and the normal fasting glucose group at 1 and 3 months. In addition, we tested for the difference in glucose levels for 0 versus 3 months and reported the p-values.

For the binary impaired fasting glucose (Yes/No) outcome, we used the generalized linear mixed effects model with the logit link function to model the proportion of IFG patients over time. The covariates structure was the same as above. The estimated proportion of IFG patients and their $95 \%$ confidence intervals were reported. Like before, we also tested for the difference in proportions for 0 versus 3 months and reported the p-values. A p-value $<0.05$ was considered statistically significant. $\mathrm{R}$ version 3.2.3 (www.r-project.org) was used for the analyses.

\section{Results}

\section{Patient population}

There were 4634 unique patients enrolled in our program from 1991-2017. A total of 2572 met the inclusion and exclusion criteria and were included in the study. While patients were enrolled from anywhere from one month to 12 months in the program we reviewed the data of the first three months of the program. The baseline characteristics of the patients are in Table 1. Among 2572 patients a total of 1176 (73.1\% female; $26.9 \%$ male) patients had IFG with fasting blood glucose between $100 \mathrm{mg} / \mathrm{dL}$ and $125 \mathrm{mg} / \mathrm{mL}$ and 1396 patients ( $77.8 \%$ female; $22.2 \%$ male) were found to having normal fasting glucose of $<100 \mathrm{mg} / \mathrm{dL}$. The groups were found to be statistically different with the average patient being older and at a greater BMI in the IFG group.

Other than appointment reminders for patients who missed two consecutive weekly appointments, there were no efforts at retention. There were 843 patients remaining in the normal fasting glucose group and 755 patients remaining in the IFG group at the end of 3 months. Patients who left the program and ones remained in the program but who missed 3 weekly visits for any reason were not included in the analysis. There were 421 patients left the program at month 3 in the IFG group.

\section{Body weight and body composition}

The change in body weight is summarized in Figure 1 . The median weight loss with a $95 \%$ confidence interval for the normal fasting glucose group was $4.2 \pm 1.3 \mathrm{kgs}$ at 1 month and $10.1 \pm 1.3 \mathrm{kgs}$ at 3 months. The total weight loss with a $95 \%$ confidence interval for the impaired fasting glucose group was $4.5 \pm 1.4 \mathrm{kgs}$ at 1 month, $10.5 \pm 1.3 \mathrm{kgs}$ at 3 months. The IFG group had a statistically higher baseline weight than the normal fasting glucose group patients. However, there was no significant difference in the weight loss between the groups at any time point. (fig 2)

The effect of the program on body fat composition is summarized in Figure 2. The average mean estimated body fat percentage by BIA at baseline was $41.5 \pm 0.9 \%$ for the IFG group and $40 \pm .8 \%$ for the normal fasting glucose group $(\mathrm{p}<0.05)$. However, there was no significant difference in the reduction in percent fat between the above two groups of patients over 12 weeks.

Fasting blood glucose 
The average blood glucose level for the IFG group of patients at baseline was $108.49 \pm 6.4 \mathrm{mg} / \mathrm{dl}$, with a statistically significant decrease to $101.8 \pm 9.41 \mathrm{mg} / \mathrm{dl}(\mathrm{p}<0.0001)$ at month 3 . The fasting blood glucose level for the group with normal fasting glucose at entry was $89.8 \pm 6.61 \mathrm{mg} / \mathrm{dl}$ with no clinically significant change at month 3 with fasting glucose levels of $91.1 \pm 8.5 \mathrm{mg} / \mathrm{dl}$.

By three months over $40 \%$ of the patients who lost 2.2-4.5 kg were able to normalize their fasting blood glucose. Within the group of patients able to lose more than $6.8 \mathrm{~kg}$ nearly $50 \%$ of patients normalized their blood glucose. (Fig 3).

Blood pressure and Lipids

The IFG group had a statistically higher systolic and diastolic blood pressure, fasting triglycerides and LDL at baseline compared to the group with normal fasting glucose.

The patients with IFG had higher median, with a $95 \%$ confidence interval, systolic blood pressure at baseline $127.2 \pm 1.1 \mathrm{mmHg}$ compared to the normal fasting glucose group patients $123.8 \pm 1.1 \mathrm{mmHg}, \mathrm{p}<0.001)$. There was significant reduction of systolic blood pressure at month 3 for both groups compared to baseline (IFG patients $121.3 \pm 1 \mathrm{mmHg} \mathrm{p}<0.001$ and normal fasting glucose patients $118.9 \pm 1 \mathrm{mmHg} \mathrm{p}<0.001)$.

The patients with IFG also had higher median, with a $95 \%$ confidence interval, diastolic blood pressure at baseline (IFG patients $81.9 \pm 2.6 \mathrm{mmHg}$ vs the normal fasting glucose group $79.5 \pm 2.4 \mathrm{mmHg}, \mathrm{p}<0.001$ ). There was a significant reduction of diastolic blood pressure at month 6 for both groups compared to baseline (IFG patients $77.3 \pm \mathrm{mmHg} \mathrm{p}<0.001$ and normal fasting glucose patients $75.5 \pm 2 \mathrm{mmHg} \mathrm{p}<0.001$ ).

The baseline total cholesterol was comparable in all patients. The triglyceride level for IFG patients was significantly decreased at month 3 by $45.2 \pm 64.2 \mathrm{mg} / \mathrm{dl}(\mathrm{p}<0.0001)$. The high-density lipoprotein cholesterol level for patients in both groups was significantly increased in association with weight loss, but without any significant difference between groups. There was a significant decrease of low-density lipoprotein at month 3 by $10.9 \pm 29.45 .18 \mathrm{mg} / \mathrm{dl}(\mathrm{p}<0.0001)$ for the IFG group and by $12.8 \pm 31.6(\mathrm{p}<0.0001)$ in the group with normal fasting blood glucose.

\section{Discussion}

Weight losses of more than $10 \%$ were observed in patients after three months of treatment and these results exceed the weight loss findings reported by the several randomized clinical trials typically report five percent weight losses. Due to the large number of subjects who did not complete the three months of clinical treatment make this estimate of weight loss less certain.

The program was effective in improving cardiovascular health parameters such as percent body fat, blood pressure (systolic and diastolic), and lipids (total Cholesterol, HDL, LDL, and Triglycerides).

We have demonstrated that this self-pay program could be a viable and economically feasible approach to delay or prevent the development of type 2 diabetes and cardiovascular diseases in patients who are overweight or obese. Our cost-effective ambulatory care model enabled physicians to partner with dietitians, nurses, and behavior therapists by utilizing group nutrition and behavioral education in a weekly schedule coordinating with time for patients to consult with physicians and dietitians individually. There is no net cost to the health care group because this is a service entirely funded by monthly patient fees. The scale of the results presented in this paper could have significant potential for far-ranging economic and social impact, helping to alleviate the growing economic burden of treating IFG, IGT, and T2DM.

The economic burden associated with T2DM and cardiovascular diseases is substantial for patients, employers, and health care systems. Research by the American Diabetes Association has estimated the cost of diagnosed diabetes in 2017 is $\$ 327$ billion, including $\$ 237$ billion in direct medical costs and $\$ 90$ billion in reduced economic productivity. Indirect costs include increased absenteeism ( $\$ 3.3$ billion) and reduced productivity while at work ( $\$ 26.9$ billion) for the employed population, reduced productivity for those not in the labor force ( $\$ 2.3$ billion), inability to work as a result of disease-related disability ( $\$ 37.5$ billion), and lost productive capacity due to early mortality (\$19.9 billion)(11). Weight management programs can reduce 
the development of T2DM if such programs can be cost-effective and efficient within our existing medical care systems. Patient-orientated interventions are the most effective in effecting positive behavioral and health outcomes (12). The impact of weight loss on medication utilization cannot be understated even though the discontinuation of medications may not have the greatest impact on overall health care costs. However, elimination of medications may be associated with reduction in potential side effects and improvement in quality of life.

We acknowledge that this study has some important limitations. It is important to note that the electronic medical records may contain some errors. Change in medications used was not captured in the electronic data entry and we may have underestimated the effects of weight loss on glucose control medications as a result. Similarly, patients on statin drugs for hypercholesterolemia also discontinued their use of drugs when low-density lipoprotein-cholesterol levels declined below the optimal levels suggested by their doctors. Prediabetes was defined by impaired fasting blood glucose values instead of oral glucose tolerance tests or hemoglobin A1c, and the lack of information on changes of medications over time. In addition, the data analysis did not examine pharmacotherapy for weight management which was added as necessary to these strategies in a small subgroup of the patient population. About five percent of patients utilized phentermine at doses of 15 to $30 \mathrm{mg}$ per day. However, pharmacotherapy was not encouraged or included in the formal program.

\section{Summary \& Conclusions:}

While the results observed were not physiologically unique, the retrospective analysis of this self-pay multidisciplinary weight management program demonstrates a cost-effective option for health care groups to incorporate into primary care. The space utilized can be clinic space that is free in evenings or weekends. Given the low cost and part-time utilization of health care professionals in the program, this program could be practically applied in ambulatory care settings to treat with prediabetes and delay or prevent onset of T2DM.

\section{Acknowledgments and statement of authors' contributions to manuscript}

VS: data collection, interpretation, and draft of the manuscript

$\mathrm{XZ}$ and CT: data analysis

DH and ZL: study design, data interpretation and draft of the manuscript

All authors read and approved the final manuscript

\section{Reference}

1. National diabetes statistics report: estimates of diabetes and its burden in the United States, 2014. In: U.S. Department of Health and Human Services CfDCaP, Atlanta, GA editor. 2014.

2. Gerstein HC, Santaguida P, Raina P, Morrison KM, Balion C, Hunt D, et al. Annual incidence and relative risk of diabetes in people with various categories of dysglycemia: a systematic overview and meta-analysis of prospective studies. Diabetes research and clinical practice. 2007;78(3):305-12.

3. Buysschaert M, Medina JL, Bergman M, Shah A, Lonier J. Prediabetes and associated disorders. Endocrine. 2015;48(2):371-93.

4. Wilson ML. Prediabetes: Beyond the Borderline. Nurs Clin North Am. 2017;52(4):665-77.

5. Ford ES, Zhao G, Li C. Pre-diabetes and the risk for cardiovascular disease: a systematic review of the evidence. J Am Coll Cardiol. 2010;55(13):1310-7.

6. Ogata S, Watanabe M, Kokubo Y, Higashiyama A, Nakao YM, Takegami M, et al. Longitudinal Trajectories of Fasting Plasma Glucose and Risks of Cardiovascular Diseases in Middle Age to Elderly People Within the General Japanese Population: The Suita Study. Journal of the American Heart Association. 2019;8(3):e010628. 
7. Dybjer E, Nilsson PM, Engstrom G, Helmer C, Nagga K. Pre-diabetes and diabetes are independently associated with adverse cognitive test results: a cross-sectional, population-based study. BMC endocrine disorders. 2018;18(1):91.

8. Rosenzweig JL, Bakris GL, Berglund LF, Hivert MF, Horton ES, Kalyani RR, et al. Primary Prevention of ASCVD and T2DM in Patients at Metabolic Risk: An Endocrine Society* Clinical Practice Guideline. J Clin Endocrinol Metab. 2019.

9. Knowler WC, Barrett-Connor E, Fowler SE, Hamman RF, Lachin JM, Walker EA, et al. Reduction in the incidence of type 2 diabetes with lifestyle intervention or metformin. The New England journal of medicine. 2002;346(6):393-403.

10. Gong Q, Zhang P, Wang J, Ma J, An Y, Chen Y, et al. Morbidity and mortality after lifestyle intervention for people with impaired glucose tolerance: 30-year results of the Da Qing Diabetes Prevention Outcome Study. The lancet Diabetes \& endocrinology. 2019;7(6):452-61.

11. American Diabetes A. Economic Costs of Diabetes in the U.S. in 2017. Diabetes care. 2018;41(5):917-28.

12. Rees S, Williams A. Promoting and supporting self-management for adults living in the community with physical chronic illness: A systematic review of the effectiveness and meaningfulness of the patientpractitioner encounter. JBI Libr Syst Rev. 2009;7(13):492-582.

\section{Figure legends}

Figure 1. Patient chart review and included in the study analysis

Figure 2: Change in Weight (kgs) and Percent Body Fat over the 3 months. Mean + standard error

Blue bar: body weight. Orange bar: \% of body fat

Figure 3: Percentage of patients with normalization of blood glucose $<100 \mathrm{mg} / \mathrm{dl}$ in relation to amount of weight lost at 3 month.

\section{Hosted file}

Table.pdf available at https://authorea.com/users/397907/articles/510643-reverseimpaired-fasting-glucose-in-overweight-and-obese-patients-participating-in-a-self-paiduniversity-weight-management-program

\section{Hosted file}

figure.pdf available at https://authorea.com/users/397907/articles/510643-reverseimpaired-fasting-glucose-in-overweight-and-obese-patients-participating-in-a-self-paiduniversity-weight-management-program 